\title{
Robust Face Detection using Convolutional Neural Network
}

\author{
Robert Yao Aaronson \\ Sch. of Comp. Sci.\& Tech \\ Jiangsu Univ. of Sci. \& Tech. \\ No. 2 Mengxi Road Jingkou \\ District Zhenjiang Prov. 212003
}

\author{
Wu Chen \\ Sch. of Comp. Sci. \& Tech \\ Jiangsu Univ. of Sci. \& Tech. \\ No. 2 Mengxi Road Jingkou \\ District Zhenjiang Prov. 212003
}

\author{
Ben-Bright Benuwa \\ Sch. of Comp. Sci. \& Comm. \\ Eng. Jiangsu Univ. Xuefu Road \\ 301 Jingkou District Zhenjiang \\ Prov. 212003
}

\begin{abstract}
Faces epitomize multifaceted dimensional meaningful visual stimuli which is a challenge for face detectors in detecting faces which is not in perfect conditions, a situation which happens often than not in real life, hence difficult developing a model for its recognition computationally. In this study, recognition rate, classification performance, estimation rate and preprocessing, and execution time of facial detection systems are improved. This is supported by the implementation of varied approaches. The face detection aspect is handled by the adaptation of Viola Jones descriptor and down-sampled by the Bessel transform which reduces feature extraction space to augment processing time. Gabor feature extractions were passed afterwards to extract thousands of facial features representing various facial deformation patterns. A deep convolutionary based Ada-boost hypothesis is carried out to select a few out of the many neurons features extracted to augment classification which are later fed into the classifier through a back-propagation algorithm. The convolutional neural network (CNN) make available for partial invariance to translation, rotation, scale, and deformation which extracts uninterruptedly larger features in a hierarchical set of layers. The results of the proposed approach were very encouraging and demonstrate superiority when compared with other state-of-the-art techniques.
\end{abstract}

\section{Keywords}

Deep learning, face detection, convolutional neural network, computer vision, Ada-boost.

\section{INTRODUCTION}

Research into automatic facial expression detection is very imperative in this modern age of scientific improvement; hence it's an extensive collection of applications including robotics, digital signs, mobile applications, and medicine. The automatic response to touching discomfort is the explicit transformation of the person face and plays a very significant task in the detection and recognition of individual emotions. It takes the occupation of an artificial intelligence system to recognize these expressions under certain circumstances, which could be spontaneous and uncontrollable in most cases.

The studies of facial detection in this contemporary times had its origin as far back in the $17^{\text {th }}$ century, much of which has been published in[1]. The work of Charles Darwin in the 19th century turns off directly impact to current date automatic facial expression detection. Darwin recognized the gist of facial detections in humans and animals, and introduced the twist patterns to facial detection recognition [2]. In the 20th century, there were massive innovations after Ekman and Friesen [3, 4] had established the Facial Action Coding System (FACS). The FACS became necessary because of the de facto standard to explain facial detections by Action Units (AUs).

Currently period facial expression (such as anger, happy, sad, surprise, fear, and neutral) researchers have more concerned and more attention than before, especially inside computer vision and pattern recognition applications. It has been described that "some robots can activate by first recognizing expressions" [5, 6]. Some other presentations of this subject are additionally basic in the behavioral sciences and prescription; for example, expression recognition is applied in medical science for intensive study and proper evaluations [6]. Many measures were developed for the enlargement of programmed facial recognition frameworks, yet they are for the most part grouped into Appearance-based and Geometric based [7]. For geometric-based approach, facial skin texture is considering instead of points and curves or deformation patterns are then extracted to form the bases of feature vectors. These vectors are to end with classifiers can be utilized prepare to distinguish location of obscure countenances. Appearance-based approaches can depend mainly on measure of the statistics of pixel standards of the face. This work done is based on geometric applications. The main noteworthy step is face detection.

A range of procedures have been planned for this duty [8-10] but incorporated skin color techniques are favored for the reason that of its robustness, straight forwardness and stronghold. The second footstep is the withdrawal of feature vectors that match up to facial deformations. A process whereby samples of different classes are in the similar way is known as unsupervised learning technique and was proposed by Eigenfaces.[11] Such Supervised learning algorithms was also proposed by Fisherfaces also $[7,12,13]$ in which facial manifestation were removed by Gabor facial appearance techniques and are better to other options because it extracts the peak information from nearby picture areas and it is invariant against, interpretation and turns. However, the major inadequacy of the Gabor characteristic means is the tremendously high computational cost.

The Bessel down sampling [14] addressed this shortfall by reducing the variety of facial components several times and by choosing a handful of Gabor wavelets from the multiple thousands via Ada-Boost based hypothesis [15]. The best classifiers that have been up to the undertaking are Support Vector Machine (SVM), [16-18] Ada-boost [19] and Neural Network [20, 21]. In this work, convolutional neural network $(\mathrm{CNN})$ as the expression classifier, it is because the fastest. The extent of the application of deep learning in this document is face detection. Face recognition several applications are use in determining it robustness, such as convenient HMI applications, but in more severe topics, such 
as surveillance software. This surveillance software scans camera video recording for faces and tracking these through facial recognition software to recognize faces. The process of detecting faces in camera footage is a difficult task, because face recognition software which is very disappointing in poor conditions. These poor situations are as appearances are not very much postured, blocked, not sufficiently bright, overuncovered, foggy, and grainy or the determination of the camera film is too low. When a face is not detected, it is difficult run through facial recognition software; hence, the demand for a high-quality face detector is elevated.

Enormously think about has been finished in the field of expression detection. However, this study is limited to articles that dived into both deep learning and face detection since the scale of this document is face detection based on CNN. The most fresh research is by[14] that proposed a deep dense face detector that was assumed to have outperformed state-of-theart face detectors. Facebook has also complete study with neural network based on face detectors, their system is called [15]. [16] also industrial a method centered on a CNN that uses selective search [17] to split an image into multiple sections. More recently, [18] carried out study constructed a face detector based on deep learning structure Caffe to find out the bearing of CNN with more training data provided. [19] also proposed CNN structural design for geometric similar based on three core apparatus that imitate the right standard of feature extraction, matching and concurrent inlier detection and model parameter estimation while being trainable from one end to the other.

The rest of the paper are arranged as follows. In Section 2 we discussed Face detection and down sampling of images. Section 3 discusses the Gabor facial feature extraction and selection. Section 4 discusses CNN classifier for face detection. The results and analysis are presented in Section 5. The conclusions of the work are drawn in section 6 .

\section{FACE DETECTION AND DOWN- SAMPLING}

Viola and Jones system [20] was adopted as in the case of other studies for the implementation of the face recognition element as shown in. Fig. 1 for classifiers and the Bessel down-sampling used to rescale of the size of the pictures and conserve the facts and perceptual value of the original image[21] as indicate in equation (1). Methods like bilinear interpolations have been utilized by quite a great deal of authors for this duty in an exact but are level to aliasing troubles [22]. (a)

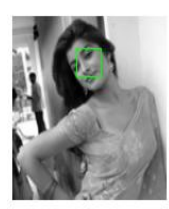

(b)

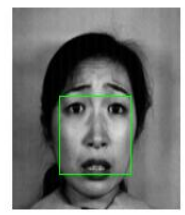

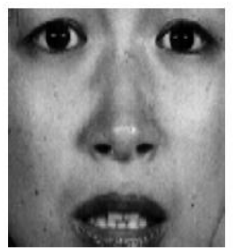

Figure 1. Sample face detections: Figure 1 (a) and (b) are sample detected images. Figure 1 (c) is a cropped image of the detected Face[23].

$$
\begin{aligned}
& y_{d}\left(m_{1}, m_{2}\right) \\
& y_{d}\left(m_{1}, m_{2}\right) \\
& y_{d}\left(m_{1}, m_{2}\right)
\end{aligned}
$$

$$
\begin{gathered}
y_{d}\left(m_{1}, m_{2}\right) \\
=\sum_{n_{1}=1}^{p} \sum_{n_{2}=1}^{q} \alpha\left(n_{1}, n_{2}\right) \\
H\left(\frac{\beta n_{1}}{p-r} m_{1}\right) H\left(\frac{\beta n_{2}}{p-f} m_{2}\right)
\end{gathered}
$$

Where the reduction values represent by $r$ and $f$ are the positive integers. $p$ and $q$ are the relevant image sizes and $p$ $\mathrm{r}$ and $\mathrm{q}_{-} \mathrm{f}$ are the required reduced size of the image; $\mathrm{n}$ is the number of low- frequency DCT coefficients, $H\left(\beta n_{1}\right)$ and $H\left(\beta n_{2}\right)$ are zero order Bessel functions, $\alpha\left(n_{1}, n_{2}\right)$ are Bessel coefficients computed from the firstorder Bessel function, $m_{1}$ and $m_{2}$ are chosen such that $0 \leq m_{1} \leq p-r$ and $0 \leq$ $m_{2} \leq p-f$. Details of these can be establish in[12].

\section{GABOR FEATURE AND FACIAL FEATURE EXTRACTION}

The 2-D Gabor filters are spatial sinusoids confined to a small area by Gaussian window, and can create to be selective for orientation, localization, and frequency as well. It is very elastic to show images by Gabor wavelets because the facts about their spatial relations are sealed in the process.

$$
\begin{gathered}
J(x, y, \emptyset, u, \delta)=\frac{1}{2 \pi \delta^{2}} \exp \left\{-\frac{x^{2}+y^{2}}{2 \delta^{2}}\right\} \\
\exp \{2 \pi i(u x \cos \emptyset+u y \sin \emptyset)\} x
\end{gathered}
$$

where $i$ is a multifarious number representing the square root of -1 . $U$ is the spatial occurrence of the band pass, $\varnothing$ is the spatial introductions of the function $\mathrm{G},(\mathrm{x}, \mathrm{y})$ require the position of light impulse in the visual field, $\delta$ is the standard deviation of 2-D Gaussian envelop. In this case, we chose five scales ranging from 0 to 4 and eight orientations ranging from 0 to 7 . In array to provide enhancement and robustness to the illumination we turned to the Gabor filter to zero DC (direct current) by the expression

$$
\begin{array}{r}
\tilde{J}(x, y, \emptyset, u, \delta)=J(x, y, \emptyset, u, \delta) \\
-\frac{1}{h}\left[\sum_{i=-n}^{n} \sum_{j=-n}^{n} J(x, y, \emptyset, u, \delta)\right]
\end{array}
$$

where, $h$ is the size of the filter, given by $h=(2 n+1)^{2}$. Fig. 2 shows the Gabor filter image and the sample points of the filtered image are coded to two bits, real bit and an imaginary bit.
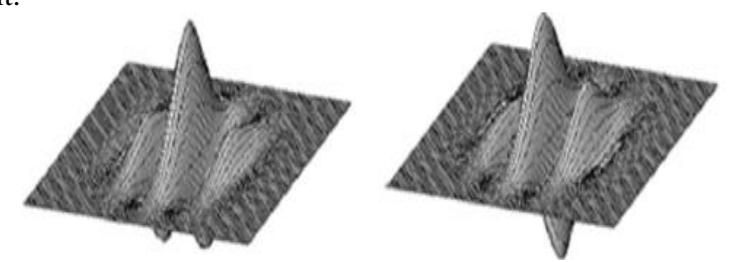

Fig2. Gabor filtered image: real (left) and imaginary (right) parts of Gabor filter in 3D[24].

It is not plausible to use all the wavelets as input to the classifier as an outcome of the large size of the Gabor wavelets, due to misclassification issues and possible system crashes. According to [25], the Ada-Boost feature reduction algorithms specially has a speed advantage hence boost 
classification processes. Subsequently the adjustment of the plan to choose some qualified a considerable lot of the wavelets. Assuming the Gabor image $I$, is collected of a total of $i \in(1,2, \ldots, N)$ appearance features. Then the image $I$, configured by $z, \mu, \quad v$ is represented as $\Phi i=\{(x n$, yn) $\} N n= \pm 1$.The positive sets $\varphi(+)$ and the negative sets $\varphi(-)$ are denoted by $\varphi(+)=\{(x n, y n)\} N n=1 \subset \mathrm{R} J \times( \pm 1)$ and $\varphi(-)=$ $\{(x n, y n)\} N n=-1 \subset R J \times( \pm 1)$ respectively, where, $x n$ is the $n$-th data sample containing $J$ features, and $y n$ is its corresponding class label. To train $\|O \mu, v(z)\|$, which is also denoted in another way as $\varphi(u, v, z)$ over a distribution $D$, the weights by determine all the feature vectors $\Phi i=\{(x n, y n)\} N n= \pm 1=\varphi(+)$ $+\varphi(-)$. This gives a threshold $\lambda$, which is the decision hyper plane. $\rho$ is computed as:

$$
\begin{gathered}
=\frac{\sum \forall}{\| \forall i \epsilon \emptyset^{(+)} D(i) \cdot \emptyset(\mu, v, z)} \\
+\mid \sum \forall i \epsilon \emptyset^{(+)} D(i) \cdot \emptyset(\mu, v, z) \| \\
+\frac{\sum \forall i \epsilon \emptyset^{(-)} D(i) \cdot \emptyset(\mu, v, z)}{\left\|\sum \forall i \epsilon \emptyset^{(-)} D(i) \cdot \emptyset(\mu, v, z)\right\|}
\end{gathered}
$$

A sample located at the positive half of $\rho$ is believe to be encouraging and usually tells the majority decision otherwise it is a pessimistic. The status is reversed if the minority of the positive instances is rather located in the positive half space. Let $\mathrm{c}$ denotes the positives and $\mathrm{p}$ is the negatives. For a given training dataset containing both encouraging and pessimistic samples, where each sample is $\left(\mathrm{S}_{\mathrm{i}}, \mathrm{y}_{\mathrm{i}}\right)$; y $\epsilon\{ \pm 1\}$ represents the corresponding class label, the feature selection algorithm is articulated as follows:

The sample distribution Do is initiated by weighing equally every training sample with the initial weight $w_{1, i}=$ $\frac{1}{2 c}, \frac{1}{2 p}$ for $y=1$ and -1 , respectively.

For $\mathrm{t}=1,2, \ldots, \mathrm{T}$ with $\mathrm{T}$ being the final restatement, execute the following perfumes

a) Normalize the weights $w_{t, i} \leftarrow \frac{w_{t, i}}{\sum_{i=1}^{N} w_{t, i}}$ where the total $\mathrm{N}$ is the number of features and $w_{t}$ the probability distribution

b) Train weak classifiers $h_{t}$ for features j which uses a particular feature and the training error $E_{t}$ estimated with value to $w_{t}$ as

$$
E_{t}=\sum_{t} w_{t, i}\left|h_{t}\left(x_{i}\right)-y_{i}\right|^{2}
$$

c) Select the hypothesis $h_{t}^{1}$ with the most discriminating information, thus the one with the least classification error $E_{t}^{1}$ on the weighted samples.

d) Compute the weight $w_{t}$ that weighs $h_{t}^{1}$ by its classification performance as

$$
w_{t}=\frac{1}{2} \ln \left[1 / E_{t}^{1}-1\right]
$$

e) Updated weight distribution and normalize.
The optimal feature selection hypothesis $H(S)$ is stated as below:

$$
H(S)=\operatorname{sine}\left[\sum_{t=1}^{T} w_{t} h_{t}^{1}\left(S_{t}^{1}\right)\right]
$$

The selected features represent samples of the facial deformation patterns of the expressive face. The datasets from which images were drawn are the ORL and YaleB databases which were partitioned into training and testing by fivefold cross validation[8].

\section{CONVOLUTIONAL NEURAL NETWORK CLASSIFIER}

$\mathrm{CNN}$ is a deep learning algorithm that is utilized as object recognition proposed by [26] which successfully succeeded [27]. The idea of a CNN is to prepare the system to copy the learning example of human beings. For instance, an infant who doesn't know of what a container is, but should see the container; severally and be informed that this is a container; the child will eventually recognize a glass when he/she sees one.

Base on therefore correlation, a $\mathrm{CNN}$ is trained by an enormous dataset. This dataset contains examples that are not focus of the exact object detection, but contain the object that needed to be located by the CNN. Figure 3 shows an example of architecture of a CNN. All the layers have exact algorithms feature extraction. Cases of highlight extraction are the complexity of a picture or what hues are there in the pictures. These layers are interrelated to each one other to combine all the feature extractions.

In the manner of training phase that will be looked when positive features are contemporary in the images and the $\mathrm{CNN}$ gives its weight to these features when they happen more frequently. When someone wants to categorize an image by a $\mathrm{CNN}$, it applies the feature extraction, and the weights that are applied the weights are calculate based on the training phase and eventually outputs consequence of the classification of an image. In addition, because some human faces can actual look as if a face of a dog, there also needs to be images supplied of objects where no faces are. Because the memory sizes of recent day computers are not sufficient to capacity all the images in its memory, the CNN performs the calculations in iterations to calculate the features. Face detection algorithms can be categorize into 3 namely cascade based, the Deformable Parts Model (DPM) based[28] and neural network based on which this model is centered. The cascade based face detectors are haar features, easy elements that explain alignment of certain pixels and a pattern of which is algorithm of [20]. The DPM is based on the constituent components that the face is fabricated centered on their geometric positions comparative to each one other. This model is robust to occlusion because when a nose and a mouth is noticed in the right geometric plane then the algorithm can pretend there should exist an eye when even the object has sun goggles on. In conclusion, a neural network based face detectors are based on self-learned features, hence can learn new patterns and this is more agile in difficult environments. 


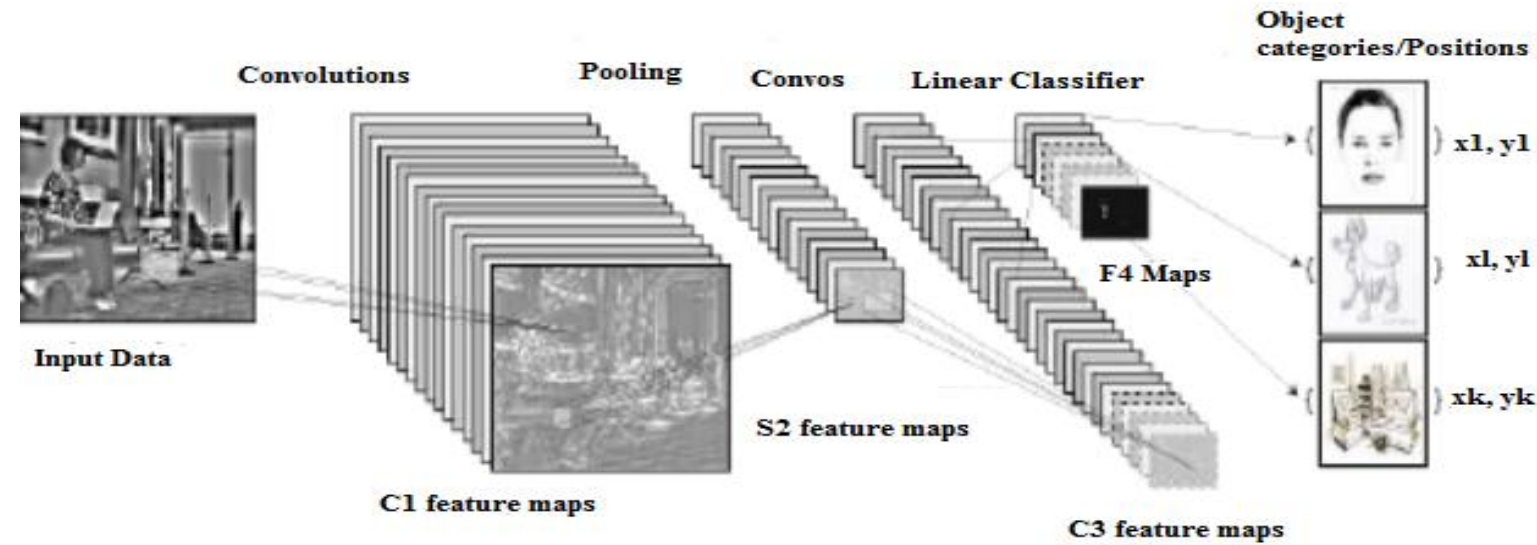

Fig3. An example of CNN [18]

The all-purpose plan of CNN is to extract trouble-free features at a high resolution, and then change them into a more complex features at a coarser resolution by sub sampling a layer by a factor of 2 , which is however a sign of the kernel size of the convolution. CNNs have been projected many years back but have not been popular in the engineering community a sign of its implementation complexities.

The preferred facial appearances are feeds into the constructed neural network to train the identifier the seven general facial expressions facial appearance. The intention of the $\mathrm{CNN}$ is train by a back-propagation algorithm that replicates its input to its output via a narrow conduit of an unseen unit. An unseen unit separate regularities from the sources of info since they are totally associated with the data sources. Each system was prepared to give the most extreme estimation of 1 for accurate facial recognition and a 0 otherwise.

Assuming the put in vectors of the network are represented by $X=\left[x_{1}, x_{2}, \ldots, x_{l}\right]^{T}$, the output layers denoted by $Y=$ $\left[y_{1}, y_{2}, \ldots, y_{k}\right]^{T}$ and the prototypical for the optimization formulated as $X: h \rightarrow Y$.

The target datasets and its addictive noise may respectively be denoted as $\left(t^{1}, t^{2}, \ldots, t^{K}\right)$ and $\mu=\left(e^{1}, e^{2}, \ldots, e^{K}\right)$. the erratic $\mathrm{K}$ represents the total patterns of the network and the corresponding vectors denoted as $V=\left(v_{1}, v_{2}, \ldots, v_{k-1}\right)$. When the training epochs are set to 1000 and the objective error is 0.001 , the training algorithm is modeled subject to some constraints as;

$$
\min _{h, w} \sum_{j=1}^{K}\left(t^{j}-y_{2}^{j}\right)^{2}
$$

Fully connected $\mathrm{CNN}$ use the formulations below to implement together with forward and backward propagations:

$$
\begin{aligned}
& x_{j}^{T+1}=\sum_{i} w_{j, i}^{T+1} x_{i}^{T} \\
& g_{i}^{T}=\sum_{i} w_{j, i}^{T+1} g_{j}^{T}
\end{aligned}
$$

Where $g_{i}^{T}$ and $x_{i}^{T}$ are respectively the gradient and the activation of unit $I$ at layer $\mathrm{T}$ and the weight connecting unit $i$ at layer $\mathrm{T}$ to unit $j$ at layer $\mathrm{T}+1$ is $w_{j, i}^{T}$. The formulations above is seen as the gradients being pooled by the lower layer units connected and the activation units being pooled by the higher layer connected to them. However, when calculating the gradients of the $\mathrm{CNN}$, the pooling strategy becomes very painful and complex to implement appropriate to the circumstance that connections leaving each one piece are not constant lane of border effects. This is problem is addressed in our model by dragging the incline from the upper layer and the CNN can discriminately be trained with back propagation algorithm. The influence updates can be done via a stochastic gradient decline as started in our formulation below;

$$
w_{i j}(t+1)=w_{i j}(t)+\mu \frac{\partial C}{\partial w_{i j}}
$$

Where $\mu$ the learning rate and $\mathrm{C}$ is is the cost function which is heavily dependent on factors like the learning type and the activation function. This study is being carried out via a supervised learning on a multiclass classification difficulty and the ordinary choices for the activation function and the cost function are the softmax and cross entropy functions respectively. The softmax function is defined as stated below;

$$
P_{j}=\frac{\exp \left(x_{j}\right)}{\sum_{k} \exp \left(x_{k}\right)}
$$

Here the total inputs to units' $\mathrm{k}$ and $\mathrm{j}$ of the same level are respectively presented by $x_{k} x_{j}$ and the unit $\mathrm{j}$, is the output that $P_{j}$ denotes the class probability. The cost function in supervised learning is the problem for multiclass classification as is well-defined as follow

$C_{r}=\sum_{j} d_{j} \log \left(P_{j}\right)-v_{j}$

Where $d_{j}$ is the target probability for the output $j$ and $v_{j}$ is the weight is then associated to the preference unit in the unseen layer. The weight is use to start at the output unit and error signals are propagated recursively value the input layer. The output detected is comparatively with the target valued which happened to be the facial image of the complete training set as a cost function for the estimated error in the targeted function in the network. The minimization error required is as stated below;

$E^{T}=\frac{1}{2} \sum_{k=1}^{K}\left(t_{k}^{T}-g_{k}^{T}\right)^{2}$

Where $E^{T}$ is the error, $t_{k}^{T}$ is well-defined as the matching target value of the detected output (the activation of the kth 
output unit) $g_{k}^{T}$. We take partial derivate to reduce the mistake with respect to each weight in the network.

$$
\begin{aligned}
\Delta v_{j i}(t+1)=-\mu & \frac{\partial E^{T}}{\partial v_{j i}}+\beta \Delta v_{j i}(t) \\
\Delta w_{k j}(t+1) & =-\mu \frac{\partial E^{T}}{\partial w_{k j}} \\
& +\beta \Delta w_{k j}(t)
\end{aligned}
$$

The learning speed $\mu$ usually between 0 and 1 but in this study, we set it to 0.9 and $\beta$ also to 0.9 and the previous term is a function of the prior weight change which mostly is approximately constant.

$$
\begin{gathered}
\frac{\partial E}{\partial v_{j i}}=\frac{\partial y_{j}}{\partial v_{j i}} \sum_{k=1}^{K}-\left(t_{k}-g_{k}\right) g_{k}(1 \\
\left.-g_{k}\right) y_{j} w_{k j} \\
\frac{\partial y_{j}}{\partial v_{j i}}=y_{j}\left(1-y_{j}\right) Z_{i}
\end{gathered}
$$

Hence,

$$
\begin{aligned}
\Delta v_{j i}=\mu \sum_{k=1}^{K} & \left(t_{k}-g_{k}\right) g_{k}(1 \\
& \left.-g_{k}\right) y_{j} w_{k j} y_{j}(1 \\
& \left.-y_{j}\right) Z_{i}
\end{aligned}
$$

The updated weights are below;

$$
\begin{aligned}
& (t+1)=w_{k j}(t)+\Delta w_{k j}(t+1) \\
& v_{j i}(t+1)=v_{j i}(t)+\Delta v_{j i}(t+1)
\end{aligned}
$$

Here $\mathrm{t}$ is the current time step, $\Delta w_{k j}$ and $\Delta v_{j i}$ is the weight adjustments and the practice is repeated from equation (13) to accomplish the desired output.

\section{RESULTS AND ANALYSIS}

The facial detection was implemented with the ORL and the Yale facial recognition databases. The first to be analyzed is ORL face repository which contains 400 face images taken from 40 different entities or subjects with each comprising of 10 facial images [29] and each respondent posed 3 or more of the seven facial detections (happy, sad, anger, fear, disgust, surprise, and neutral). More so, certain images are snappy at different times with different facial expressions and details, under varying light intensity, images captured homogenously against dark and grey surroundings of the individuals in an erect frontal position.

Every image was resized using Matlab codes to $32 \times 32$ image matrixes to ensure sufficient data in the estimation of Gaussian models and for covariance matrices with positive definite. 4 images each was randomly selected constituting 160 images for training and the rest preserved for testing and the trial performed using fivefold cross validation to obtain an standard identification rate. The images were randomly corrupted manually by an isolated building block image at random locations. The detection accuracies at dissimilar or various occlusion levels are revealed in table 2 . The second is the Yale Face Database that contains a sum of 165 ancient images of 15 subjects. The images taken under varying light intensity conditions were normalized to a size of $32 \times 32$ and the database was at random distributed into two halves. There are 11 images per subject that exhibited at least show one of the six facial expressions One set containing pictures of 130 for every individual or subject utilized as the preparation and the staying set utilized for testing. We recorded a normal acknowledgment rate of $96.83 \%$ in ORL and $92.22 \%$ in Yale on Intel(R) Core(TM) i5-2450M @ $2.50 \mathrm{GHz}$ (4 CPUs) - 2.5 $\mathrm{GHZ}$ and $8.0 \mathrm{GHz}$ RAM computer running on Windows 7 Ultimate 64-bit (6.1, Build 7601) (see Tables 1 and 2 for the confusion matrices). The best recognitions were detected in happy, sad and disgust, where we obtained almost $100 \%$ in the ORL database indicating the best results.

Generally, the presentation of neutral weakest was about $92.27 \%$ in ORL and 87.12 in Yale. The consequences indicate that the deformations of the muscles approximately the mouth and the eyes are the mainly dependable determinants for automatic facial detections. This records why detection in the neutral face is unfortunate. Thus the enlarged in these influence deformations increases the accurateness of automatic recognitions. The execution time for a pixel of size $100 \times 100$ of the proposed method is significantly less as compared to the other classifiers. Fig. 5 shows the relative presentation of implementation time with other neural network classifiers.

Table 1: Confusion matrix of 4-class outward facial expression recognition on Yale.

\begin{tabular}{|l|l|l|l|l|}
\hline $\begin{array}{l}\text { Feature } \\
(\%)\end{array}$ & Happy & Surprise & Sad & Neutral \\
\hline Happy & 0 & 4.09 & 10.05 & 87.12 \\
\hline Surprise & 0 & 5.03 & 87 & 38.78 \\
\hline Sad & 98.2 & 0.9 & 0 & 1.93 \\
\hline Neutral & 0 & 98.54 & 0.79 & 1.04 \\
\hline \multicolumn{5}{|c|}{ Average Recognition $=\mathbf{9 2 . 2 2 \%}$} \\
\hline
\end{tabular}

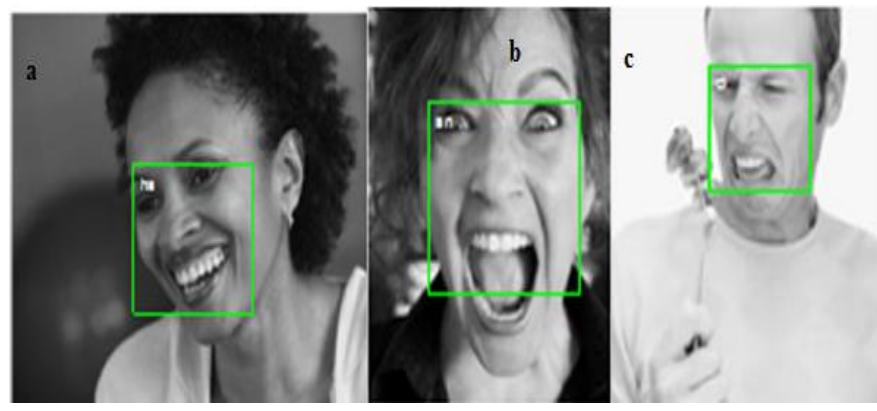

Fig 4 Examples facial expression detections: happy (a), sad (b) and disgust (c) 
Table 2: Confusion matrix of 7-class facial expression recognition on ORL.

\begin{tabular}{|l|l|l|l|l|l|l|l|}
\hline & Happy (\%) & Fear $(\%)$ & Neutral $(\%)$ & Disgust (\%) & Sad (\%) & Anger (\%) & Surprise (\%) \\
\hline Happy & 0 & 1.13 & 92.27 & 0 & 4.33 & 2.37 & 0 \\
\hline Fear & 0 & 1.31 & 3.86 & 0 & 94.2 & 0.98 & 0 \\
\hline Neutral & 0 & 96.12 & 0 & 1.85 & 0.97 & 0 & 1.16 \\
\hline Disgust & 0 & 1.14 & 0.24 & 0 & 0 & 96.14 & 0 \\
\hline Sad & 0 & 0 & 1.28 & 99.94 & 0 & 0.64 & 0.32 \\
\hline Anger & 99.74 & 0.09 & 0 & 0 & 0 & 0 & 0 \\
\hline Surprise & 0 & 0.3 & 0.1 & 0.07 & 0 & 0 & 99.88 \\
\hline
\end{tabular}

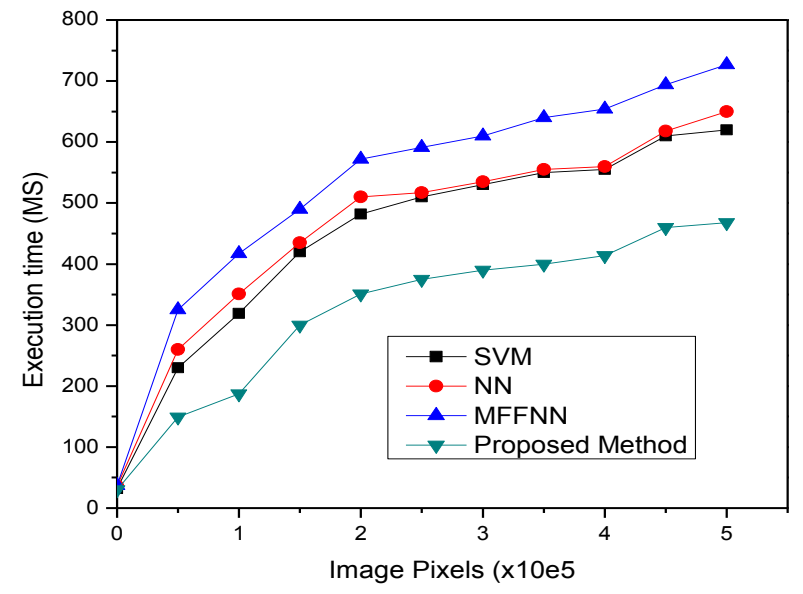

Fig 5. The execution time of the proposed method against other classifiers

Table 3: Analysis of Recognition rates in different methods on ORL database.

\begin{tabular}{|c|c|}
\hline Method/Classifier & Rate \\
\hline 2D-DLPP & 95.91 \\
PCA \& NN & 93.72 \\
RDA & 96.7 \\
DDFD & 91.79 \\
MFFNN & 96.81 \\
RFDCNN (proposed method) & $\mathbf{9 7 . 8 9}$ \\
\hline
\end{tabular}

Table 4: Analysis of Recognition rates in different methods on Yale database.

\begin{tabular}{|c|c|}
\hline Method/Classifier & Rate \\
\hline SVM & 89.50 \\
NN & 84.82 \\
MFFNN & 91.52 \\
RFDCNN (proposed method) & $\mathbf{9 6 . 4 7}$ \\
\hline
\end{tabular}

\section{CONCLUSION AND RECOMMENDATIONS}

In this paper, a novel approach to facial expression recognition that adopts many innovative techniques to advance the recognition rate and also, execution time of face recognition frameworks is created. Confront recognition was completed by the utilization of Viola-Jones descriptor. Detected countenances were down examined by the Bessel change. This approach decreased the picture measurements and protected the perceptual nature of the first picture.

An Ada Boost based algorithm planned to choose a couple of many Gabor wavelets from the few a huge number of the removed elements to lessen the computational cost and to evade misclassification too. The selected components were nourished into an all-round planned multi-layered $\mathrm{CNN}$ classifier. The change that this strategy exhibits propose that moving past profound learning is deserving of further adventure. The system is hence prepared with test datasets from both ORL and Yale facial databases. The rest of the datasets from the two databases were utilized to test for the framework. The execution time of $100 \times 100$ pixel size is 14.5 ms; normal acknowledgment rate in ORL database is $96.89 \%$ and that in Yale is $92.27 \%$.

The proposed strategy is contrasted and a few techniques and the execution are exceptional. The after effects of the review additionally demonstrate that programmed expression recognitions are exceptionally precise in amazement, sickens and glad; $100 \%$. Soft expressions like miserable, dread and impartial have brought down exactness.

Nevertheless, dread can be extremely precise when it is at the pinnacle since correctness's in recognitions largely rely upon the greatness of facial disfigurements around the mouth and eyes. To progress towards $100 \%$ productivity we trust the advancement of common databases would be of more help since numerous fake databases have many confounded situations among outward appearances in dismal, unbiased and gentle outrage. Again future changes of acknowledgment correctness's will take a gander at the likelihood of expanding the quantity of concealed neurons to expressions that recorded lower values. Improvements of recognition correctness will look at the probability of accumulative the number of unknown neurons to expressions that recorded lower values. In future, we would like to incorporate sparse representation with deep machine learning [30] into the structure of $\mathrm{CNN}$ to improve the classification performance of the structure. 


\section{REFERENCES}

[1] 1. Schmitter, A.M. and J. Montagu, The Expression of the Passions: The Origin and Influence of Charles LeBrun's" Conférence sur l'expression générale et particulière". 1996, JSTOR.

[2] Fridlund, A.J., Human facial expression: An evolutionary view. 2014: Academic Press.

[3] Ekman, P. and W.V. Friesen, Facial action coding system. 1977.

[4] Ellsworth, P.C. and C.A. Smith, From appraisal to emotion: Differences among unpleasant feelings. Motivation and Emotion, 1988. 12(3): p. 271-302.

[5] Bruce, V., What the human face tells the human mind: Some challenges for the robot-human interface. Advanced Robotics, 1993. 8(4): p. 341-355.

[6] Brockhausen, P., T. Joachims, and K. Morik, Combining statistical learning with a knowledge-based approach. 1999, Universitätsbibliothek Dortmund.

[7] Belhumeur, P.N., J.P. Hespanha, and D.J. Kriegman, Eigenfaces vs. fisherfaces: Recognition using class specific linear projection. IEEE Transactions on pattern analysis and machine intelligence, 1997. 19(7): p. 711720.

[8] Wu, J., et al., Fast asymmetric learning for cascade face detection. IEEE Transactions on Pattern Analysis and Machine Intelligence, 2008. 30(3): p. 369-382.

[9] Delac, K., M. Grgic, and S. Grgic, Independent comparative study of PCA, ICA, and LDA on the FERET data set. International Journal of Imaging Systems and Technology, 2005. 15(5): p. 252-260.

[10] Tan, X., et al., Face recognition from a single image per person: A survey. Pattern recognition, 2006. 39(9): p. $1725-1745$

[11] Turk, M. and A. Pentland, Eigenfaces for recognition. Journal of cognitive neuroscience, 1991. 3(1): p. 71-86.

[12] Al Daoud, J.E., Enhancement of the face recognition using a modified Fourier-Gabor filter. Int. J. Advance. Soft Comput. Appl, 2009. 1(2).

[13] Mahmoud, S.A. and W.G. Al-Khatib, Recognition of Arabic (Indian) bank check digits using log-Gabor filters. Applied Intelligence, 2011. 35(3): p. 445-456.

[14] Farfade, S.S., M.J. Saberian, and L.-J. Li. Multi-view face detection using deep convolutional neural networks. in Proceedings of the 5th ACM on International Conference on Multimedia Retrieval. 2015. ACM.

[15] Taigman, Y., et al. Deepface: Closing the gap to humanlevel performance in face verification. in Proceedings of the IEEE Conference on Computer Vision and Pattern Recognition. 2014.
[16] Girshick, R., et al. Rich feature hierarchies for accurate object detection and semantic segmentation. in Proceedings of the IEEE conference on computer vision and pattern recognition. 2014

[17] Van de Sande, K.E., et al. Segmentation as selective search for object recognition. in Computer Vision (ICCV), 2011 IEEE International Conference on. 2011 IEEE.

[18] Van Kleef, J., Towards Human-like Performance Face Detection: A Convolutional Neural Network Approach. 2016.

[19] Rocco, I., R. Arandjelović, and J. Sivic, Convolutional neural network architecture for geometric matching. arXiv preprint arXiv:1703.05593, 2017.

[20] Viola, P. and M.J. Jones, Robust real-time face detection. International journal of computer vision, 2004. 57(2): p. 137-154.

[21] Sridharan, M., et al., NVGRE: Network virtualization using generic routing encapsulation. IETF draft, 2011.

[22] Muñoz, A., T. Blu, and M. Unser, Least-squares image resizing using finite differences. IEEE Transactions on Image Processing, 2001. 10(9): p. 1365-1378.

[23] Owusu, E., Y. Zhan, and Q.R. Mao, An SVM-AdaBoost facial expression recognition system. Applied intelligence, 2014. 40(3): p. 536-545.

[24] Owusu, E., Y. Zhan, and Q.R. Mao, A neural-AdaBoost based facial expression recognition system. Expert Systems with Applications, 2014. 41(7): p. 3383-3390.

[25] Shen, L. and L. Bai. AdaBoost Gabor feature selection for classification. in Proc. of Image and Vision Computing NewZealand. 2004

[26] Vaillant, R., C. Monrocq, and Y. Le Cun, Original approach for the localisation of objects in images. IEE Proceedings-Vision, Image and Signal Processing, 1994. 141(4): p. 245-250.

[27] Fukushima, K. and S. Miyake, Neocognitron: A selforganizing neural network model for a mechanism of visual pattern recognition, in Competition and cooperation in neural nets. 1982, Springer. p. 267-285.

[28] Felzenszwalb, P., D. McAllester, and D. Ramanan. A discriminatively trained, multiscale, deformable part model. in Computer Vision and Pattern Recognition, 2008. CVPR 2008. IEEE Conference on. 2008. IEEE.

[29] Samaria, F.S. and A.C. Harter. Parameterisation of a stochastic model for human face identification. in Applications of Computer Vision, 1994., Proceedings of the Second IEEE Workshop on. 1994. IEEE.

[30] Benuwa, B.B., et al. A Review of Deep Machine Learning. in International Journal of Engineering Research in Africa. 2016. Trans Tech Publ. 